\title{
Pain Management Therapy: The Benefits of Compounded Transdermal Pain Medication
}

\section{Andrea Branvold ${ }^{*}$ and Maria Carvalho}

PCCA, 9901 South Wilcrest Dr., Houston, TX 77099-5132, USA

*Corresponding author: Andrea Branvold, MS, RPh, PCCA, 9901 South Wilcrest Dr., Houston, TX 77099-5132, USA, Tel: 2819336948; Fax: 281.933.6627; E- mail: abranvold@pccarx.com

Received date: November 5, 2014; Accepted date: November 20, 2014; Published date: November 27, 2014

Copyright: ( 2014 Branvold A, et al. This is an open-access article distributed under the terms of the Creative Commons Attribution License, which permits unrestricted use, distribution, and reproduction in any medium, provided the original author and source are credited.

\begin{abstract}
The high worldwide incidence of both acute and chronic pain leads to widespread consequences for both the healthcare system and the economy. First-line pain treatment options are typically oral pain medications; however, concerns regarding side effects, prescription drug abuse, risk of overdose, patient non-adherence to treatment regimens, and lack of efficacy in certain conditions provide a number of challenges for both healthcare providers and patients. As a result, healthcare providers have become increasingly interested in new ways to manage pain and develop customized treatment plans for their patients. Compounded transdermal pain medication may offer benefits such as customizable dosages and formulations, the ability to combine multiple drugs with various mechanisms of action, the likelihood of lower systemic absorption with minimization of side effects, more convenience and consequent improved adherence to treatment regimens, and minimization of risk of abuse and addiction. This review offers an overview of examples to provide evidence of the benefits of transdermal compounded pain medication in the treatment of a number of pain-related conditions, including neuropathic, musculoskeletal, arthritis-related, and postoperative pain, among other conditions.
\end{abstract}

Keywords: Pain management; Acute pain; Chronic pain; Transdermal medications; Compoundedmedications

\section{Introduction}

Pain is a highly complex concept that has a different effect on and unique perception for each individual pain sufferer. This multifaceted phenomenon involves physical, cognitive, and emotional factors and thus requires an individualized treatment plan [1]. Pain may be a symptom that results from inflammation or changes to the nervous system due to injury or disease and can also be a primary disease itself [2]. Pain affects more Americans than diabetes, heart disease, and cancer combined and is cited as the most common reason that Americans access the healthcare system [3]. A survey by the National Institutes of Health found that $21 \%$ to $30 \%$ of adults reported pain lasting more than 24 hours during the course of a given month, and the most commonly reported types of pain were low back pain $(27 \%)$, severe headache or migraines (15\%), neck pain (15\%), and facial ache or pain (4\%) [2]. As a result of the high number of pain sufferers, nearly 260 million prescriptions for opioid painkillers were written in 2012 [4].

\begin{tabular}{|l|}
\hline Challenges with oral pain medication \\
\hline Side effects \\
\hline Gastrointestinal \\
\hline Renal \\
\hline Hepatic \\
\hline Cardiovascular \\
\hline Sedation and decreased cognition \\
\hline
\end{tabular}

\begin{tabular}{|l|}
\hline Abuse and addiction \\
\hline Administration difficulties \\
\hline Non-compliance \\
\hline Lack of Efficacy \\
\hline
\end{tabular}

Table 1: Challenges with oral pain medication.

Chronic pain refers to pain conditions that persist due to a disease or injury (sometimes even after the injury is healed) because of pain signals that remain active in the nervous system [5]. This type of pain may affect a patient for weeks, months, or even years and may produce both physical effects, such as tense muscles, limited mobility, a lack of energy, and changes in appetite, as well as emotional effects, such as depression, anger, anxiety, and fear of re-injury [5]. Chronic pain affects more than 1.5 billion people worldwide, including nearly 100 million American adults, and results in a national economic burden of up to $\$ 635$ billion per year in medical treatment and lost productivity in the workforce due to work disability and medical appointments [1]. Chronic pain is the most common cause for longterm work disability [3]. The most common chronic painrelated conditions include severe headaches; musculoskeletal injuries, such as back or neck pain; arthritis; cancer; neuropathic pain; and psychogenic pain [5].

Acute pain is the result of an injury or disease that typically begins suddenly and can be sharp in quality [5]. It can range from mild to severe in intensity, lasting for just a moment to weeks or even months. Upon treatment, acute pain will cease when the underlying cause of the pain, such as the disease or injury, is cured. Acute pain commonly results from events including minor injuries, surgery, broken bones, dental work, burns or cuts, or labor and childbirth. While it is known 
that millions suffer from acute pain, the exact number of acute pain sufferers is difficult to obtain due to acute pain's temporary nature and the failure many pain sufferers to report or seek treatment for their condition $[2,3]$.

Each pain sufferer's experience of pain is unique, requiring a pain management plan customized to the individual's conditions and needs. Pain management plans may involve a combination of medicines that may take a variety of forms. First-line treatments typically include oral medications, such as non-steroidal antiinflammatory drugs (NSAIDs), muscle relaxants, antidepressants, and anticonvulsants for nerve pain, and may also include narcotics. Significant patient challenges associated with oral pain medication have led to a growing interest in the use of compounded transdermal pain medications as beneficial alternative treatment options.

\section{Discussion}

\section{Patient and prescriber challenges with oral pain medication}

The most commonly prescribed pain medications are typically orally administered. However, growing concerns regarding the prescription of oral pain medication include the serious side effects associated with oral medication and the rise in prescription drug abuse and addiction (Table 1). Many patients are prescribed multiple oral medications to treat various types of pain, leading to patients administering many capsules and pills throughout the day, which often becomes a daunting task that can lead to non-compliance [6].

\section{Side effects}

Common oral medications prescribed for the management of acute or chronic pain include NSAIDs, acetaminophen, narcotics, muscle relaxants, tricyclic antidepressants, and anticonvulsants, such as gabapentin. While these oral medications may be effective in treating pain, there are challenges associated with long-term use of these medications for both the prescriber and patient (Table 2).

Non-Steroidal AntiInflammatory Drugs (NSAIDS): Oral NSAID use presents the risk of a number of potential side effects, particularly with chronic use [7]. While some less serious side effects, such as nausea, are common, of particular concern are the potentially severe side effects in the gastrointestinal (GI) tract, including GI bleeding, perforation, and intestinal toxicity. Long-term exposure to oral NSAIDs has been associated with ulcer rates of 10 to $30 \%$, serious ulcer-related complications in 1 to $2 \%$ of patients, and an increased risk of lower GI bleeding [8]. An estimated 100,000 patients are hospitalized annually in the US as a result of NSAID-related GI complications, with mortality rates of up to $5 \%$ [8]. To reduce the risk of GI toxicity from NSAIDs, the addition of gastroprotective agents such as $\mathrm{H} 2$ receptor antagonists and proton pump inhibitors (PPIs) are suggested, but PPIs have been shown to fail to protect against lower GI complications and have their own potential for adverse events [8]. In addition, over $30 \%$ of patients prescribed PPIs in addition to pain medication are non-compliant with their treatment regimens [8].

Additional concerns with the chronic use of oral NSAIDs are the increased risk of nephrotoxicity, cardiovascular adverse events, and cartilage degeneration. Perneger et al. reported that the heavy use of oral NSAIDs leads to 4 -fold greater risk of end-stage renal disease [9].
Other renal adverse events associated with oral NSAID use include renal papillary necrosis, acute deterioration of renal function, acute interstitial nephritis, hyperkalemia, and sodium and fluid retention [7]. In addition, NSAID exposure has been associated with higher risks of cardiovascular complications, such as myocardial infarction, heart failure, and hypertension. Chronic NSAID use has also been linked to increased cartilage degeneration resulting in the need for joint replacements to occur more frequently or more quickly [10]. Like many other side effects, these events have been connected to the duration of NSAID exposure [8].

Acetaminophen: Another commonly used oral pain medication is acetaminophen, which is a popular choice due to its ease of accessibility and low cost. However, a main concern of chronic acetaminophen use is overuse and toxicity [11]. Acetaminophen is the most commonly reported toxic ingestion in the United States reaching over 165,000 exposures in 2005, including approximately 67,000 toxic ingestions of acetaminophen alone. Overdose of acetaminophen is known to cause kidney damage, with renal insufficiency occurring in 1 to $2 \%$ of patients who experience acetaminophen overdose. In addition, acetaminophen overdose is a leading cause of acute liver failure in the US [12]. Acetaminophen poisoning accounts for approximately $42 \%$ of acute liver failure cases and one-third of the deaths associated with liver failures. Unfortunately, these unintentional overdoses are often not recognized or treated until after symptoms have developed, requiring that both patients and healthcare providers be highly cautious with chronic acetaminophen use.

Narcotics (Opioids): Narcotics are another class of oral medications commonly prescribed for the management of acute or chronic pain, but like other oral pain medications, these also come with concerns of side effects. Common side effects associated with oral narcotics include nausea (affecting approximately 25\% of patients) and constipation [13]. Of more serious concern are central nervous system (CNS) effects, including sedation and decreased cognition. The incidence of sedation reported with narcotic use is between 20 and $60 \%$, most commonly reported in conjunction with initiation of therapy or dose increases. Cognitive changes, which can lead to cognitive dysfunction, the inability to concentrate, persistent confusion, and delirium, are also a serious concern. Consequently, regular monitoring of patients using narcotics for pain management is necessary to evaluate the safety and efficacy of this treatment option. For some patients, these cognitive and sedative effects make regular treatment with narcotics too inconvenient.

In addition to narcotics alone, treatment options that combine narcotics with acetaminophen, such as Vicodin and Percocet, are commonly prescribed. These drugs also carry the risks of side effects associated with narcotics, as well as the increased risk of liver toxicity associated with acetaminophen use. In fact, the US Food and Drug Administration (FDA) recently banned the production of narcotic/ acetaminophen products with doses of acetaminophen greater than $325 \mathrm{mg}$ and has required the addition of liver toxicity drug label warnings for these products in order to temper the incidence of druginduced liver toxicity caused by these medications [14].

Muscle Relaxants and Other Therapies: In some cases, such as in patients with chronic low back pain, antispasmodic muscle relaxants are prescribed, although these are generally not considered first-line therapies. Common muscle relaxants, including cyclobenzaprine and carisoprodol, are often prescribed either in conjunction with acetaminophen or NSAIDs or after first-line therapies have been shown to be ineffective or not tolerated [15]. The main drawback of 
these therapies is their potential for CNS adverse effects, such as dizziness or drowsiness. Another common pain therapy is gabapentin, an anticonvulsant drug, which is generally used to treat epilepsy but has been prescribed for pain management for neuropathic conditions and postoperative recovery $[16,17]$. Like muscle relaxants, gabapentin is associated with CNS side effects, including increased sedation and dizziness. Tricyclic antidepressants, such as amitriptyline, have also been prescribed for the management of neuropathic pain and are considered first-line therapy for certain conditions, such as diabetic neuropathy [18]. As with the other alternative treatments mentioned, tricyclic antidepressants are associated with CNS side effects as well as constipation, dry mouth, and tachycardia [18].

\begin{tabular}{|l|l|}
\hline Oral Pain Medication & Common Side Effects \\
\hline NSAIDS & $\begin{array}{l}\text { GI toxicity and complications such as bleeding, } \\
\text { perforation, and ulcers; nephrotoxicity, } \\
\text { cardiovascular disease, and cartilage } \\
\text { degeneration. }\end{array}$ \\
\hline Acetaminophen & $\begin{array}{l}\text { Toxic ingestion: renal insufficiency and acute liver } \\
\text { failure }\end{array}$ \\
\hline Narcotics (Opioids) & $\begin{array}{l}\text { CNS effects such as sedation and decreased } \\
\text { cognition }\end{array}$ \\
\hline Muscle relaxants & CNS effects such as dizziness and drowsiness \\
\hline Tricyclic antidepressants & $\begin{array}{l}\text { CNS effects such as constipation, dry mouth, and } \\
\text { tachycardia }\end{array}$ \\
\hline
\end{tabular}

Table 2: Common side effects of oral pain medication.

\section{Abuse and addiction}

A major concern with the use of prescription oral pain medication is the growing problem with addiction, abuse, and overdose, particularly with the use of opioid painkillers. The US Centers for Disease Control and Prevention (CDC) reports that there has been at least a 10 -fold increase in opioid painkiller prescriptions over the past 2 decades [19]. Conflicting views on the type and dosing of prescription painkillers necessary for patients suffering from pain has contributed to this increase [20]. The highly addictive nature of opioid drugs and this rapid increase in prescriptions has led to serious problems with prescription painkiller addiction and abuse. Drug overdoses have surpassed motor vehicle crashes as the major cause of unintentional death in the US [21]. Currently, 46 people a day die from prescription drug overdoses in the US [4]. In 2010, opioid analgesics accounted for nearly $60 \%$ of drug overdose deaths, which is higher than the number of deaths caused from overdose of all illicit drugs combined, including heroin and cocaine [22]. This number has increased from $30 \%$ of drug overdoses in 1999, highlighting the urgency of these issues [22]. Beyond the health implications of this problem, such abuse also has serious economic consequences. The costs of prescription opioid abuse have been estimated to be over $\$ 55$ billion in the US alone, accounting for lost work productivity, health care costs, and criminal justice costs [23].

\section{Administration challenges}

Many pain management plans, particularly in the treatment of chronic pain, rely on polypharmacological approaches to provide the patient with the most effective, long-term pain relief [24]. These plans often combine a series of oral medications targeting different mechanisms of pain relief. As a result, patients may have to administer multiple pills at different times of day, which can become taxing and confusing for some patients. The World Health Organization cites "complexity of regimen" as 1 of the 4 main reasons for lack of patient adherence to treatment regimens [25]. The greater number of pills per dose or doses per day, as well as strict label restrictions on food and liquid intake surrounding drug administration contribute to lower patient adherence to treatment plans. A study by Claxton et al. found that patient compliance was significantly higher for once-daily medications than for 3-times-daily or 4-times-daily medications [26]. This problem can be further complicated by the addition of multiple medications that require different dosing schedules. Common reasons patients cite for not adhering to their medication regimens include forgetfulness, other priorities, and the decision to omit doses [27]. Thus, non-adherence to medication regimens can be a serious issue among patients being treated for chronic pain.

\section{Lack of efficacy}

In most cases, patients rely on their primary care physicians for the treatment of pain-related conditions; however, many primary care physicians feel that they have had insufficient training regarding pain treatment [1]. This problem results in errors in prescribing the correct type of medication for the patient's pain. For example, a recent study by Mafi et al. showed that physicians have increasingly been prescribing narcotics and other second-line therapies for chronic back pain over first-line therapies outlined in published guidelines, such as NSAIDs and acetaminophen [28].

\section{Potential prescriber challenges}

Healthcare providers are faced with increased challenges involving the prescribing of pain medication for their patients due to issues with overprescribing, monitoring by medical boards, and liability. The CDC reports that there has been at least a 10 -fold increase in the prescribing of opioid painkillers over the past 2 decades [19]. This increase is partially due to the growing problem of overprescribing by healthcare providers, some of which is due to abuse of medical privileges, but has also been connected to conflicting views on the type and dosing of prescription painkillers necessary for patients suffering from pain [20].

Due to issues with abuse of opioids, there have been increased regulatory efforts to limit the prescribing of oral opioids. The US Drug Enforcement Administration (DEA) recently moved hydrocodone to a more restrictive classification, limiting prescriptions of drugs containing this active ingredient to 90 -day quantities, thus, requiring patients to revisit their healthcare provider to obtain a new prescription following the 90-day time limit [29]. The health insurance company Blue Cross Blue Shield has recently issued limitations on the coverage of prescription opioids, such as Vicodin and Percocet. Under the new policy, most patients will only qualify for a 15 day supply of these drugs with a 15-day refill before requiring approval for additional refills of these prescriptions [30].

With the increase in regulation of these prescription painkillers, as well as the concern for patient injury or death resulting from the abuse of these drugs, healthcare providers are more cautious about prescribing these types of painkillers and may be looking for alternatives to treat their patients, particularly those suffering with chronic pain. Physicians are more conscious about the potential for liability relating to prescribing painkillers that may present risk for addiction, especially for chronic pain. They are also aware of the 
Page 4 of 8

increased monitoring of prescription writing as this issue comes under further scrutiny due to growing patient safety problems. As a result, the use of transdermal pain medication may be considered an alternative to oral painkillers in order to reduce concerns of patient abuse and prescriber liability.

\section{Compounded transdermal pain medications}

Currently, pain medications are dominated by the pharmaceutical industry but given the increasing concerns regarding side effects, administration challenges, and addiction and abuse of oral painkillers, interest in the use of transdermal pain medication as an alternative therapeutic option for pain management has grown. Compounded transdermal medications may avoid some of the challenges associated with oral pain medication as well as commercially available patches, while providing added benefits of being customizable to a patient's specific needs. Other benefits of transdermal administration include convenience and ease of administration, improved patient compliance, and allowing for less frequent dosing [31].

Compounding is described in Chapter 795 of the United States Pharmacopeia (USP) as "the preparation, mixing, assembling, altering, packaging, or labeling of a drug, drug-delivery device, or device in accordance with a licensed practitioner's prescription, medication order, or initiative" [32]. The practice of compounding dates back to ancient times with early "druggists" or chemists and has continued to be practiced throughout history [33]. Compounding was a main part of a pharmacist's profession in the early 20th century and accounted for approximately $60 \%$ of the medications provided to patients in the mid-20th century [33]. However, with the introduction of penicillin and the subsequent trend towards mass-produced drugs, compounding declined significantly, now accounting for only $1 \%$ of the drugs prescribed today [33]. Interest has been renewed in recent years as pharmacists realize they can better meet the individualized needs of many patients through the practice of compounding.

Although compounded medications may have unique formulations, they are prepared under the guidance of the USP, often through the combination of active pharmaceutical ingredients (APIs) utilized in FDA-approved drugs. Established in 1820, the USP sets standards for prescription drugs and the practices of pharmacists in the US [34]. Many documents have been issued and periodically revised by the USP outlining guidelines for practices related to pharmaceutical compounding, most notably Chapter 795, Pharmaceutical Compounding-Nonsterile Preparations, which discusses proper compounding environment and equipment, ingredient selection, appropriate dosing, processes, quality control, and patient counseling [32]. In the US, compounding-specialist pharmacies are common and pharmacists have the expertise to prepare transdermal pain medications in accordance with current standards. Additional training on compounding is available to US pharmacists and technicians nationwide in the format of courses and symposiums [35].

\section{History of compounded transdermal agents}

Transdermal pain compounds are typically prepared by using either a single or combination of multiple APIs in a specially designed compounding base. These bases are transdermal drug delivery systems designed to deliver 1 or more drugs through the skin. The first widely recognized compounded transdermal base was Pluronic Lecithin Organogel (PLO), which is an oil and aqueous emulsion introduced as a drug delivery vehicle in the 1990s [36]. The development of this base was highly important for the progress of compounding transdermal medication; however, it is considered first generation and more advanced versions of transdermal bases have been introduced, such as Lipoderm ${ }^{\circ}$, which has shown improvements in consistency and stability [37,38]. Lipoderm ${ }^{\circ}$ has been shown to successfully deliver 4 pain medications, including ketamine hydrochloride $(\mathrm{HCl})$, gabapentin, clonidine $\mathrm{HCl}$, and baclofen, simultaneously into human skin [39].

\section{Benefits of transdermal pain medication}

Utilizing transdermal compounds for pain management presents opportunities to customize regimens to meet the challenges of treating pain. Potential benefits include side effect minimization, combination of multiple active ingredients in a single formulation (thereby providing greater convenience and potentially better efficacy than single-ingredient products), application of the medication directly to the site of pain, easy titration to meet individual patient needs, lower systemic absorption, and improvement of patient adherence to treatment regimens (Table 3 ).

\begin{tabular}{|l|}
\hline Potential benefits of transdermal compounded medication \\
\hline Customizable dosages, formulations, and drug combinations \\
\hline Ability to combine multiple drugs with various mechanisms of action \\
\hline Application directly at the site of pain \\
\hline Potential for less systemic absorption and minimization of side effects \\
\hline More convenience and better adherence to treatment regimen \\
\hline Easy adjustment or titration to meet patient needs \\
\hline Minimization of abuse and addiction risk \\
\hline
\end{tabular}

Table 3: Potential benefits of transdermal compounded medication.

Customizable dosages, formulations, and drug combinations: When a healthcare provider orders a compounded transdermal pain prescription, the APIs and their percentages can be tailored to a patient's individual needs. A number of different APIs used transdermally to treat various types of pain, such as NSAIDs, anesthetics, anticonvulsants, hypotensive agents, and muscle relaxants, can be combined at appropriate dosages all in a single formulation. This flexibility allows for a single prescription to target multiple receptors involved with pain signal transmission. Once a combination of pain APIs is determined, the appropriate transdermal base is chosen. The drugs are incorporated into the base at specific concentrations, and the final preparations are dispensed as any other prescription with specific dosing instructions for the patient. (For an example of how to prescribe a compounded transdermal pain prescription for otherwise healthy adult patients (Table 4). This provides an example of the type of compounded pain prescription that may be ordered from a compounding pharmacy [40]).

\begin{tabular}{|l|}
\hline Writing a compounded transdermal pain prescription \\
\hline $\begin{array}{l}\text { Include percentages of active pharmaceutical ingredients (APIs) and } \\
\text { transdermal base. }\end{array}$ \\
\hline Example of classes of drugs used typically for neuropathic pain: \\
\hline API (anesthetic)\%
\end{tabular}


Page 5 of 8

\section{API (anticonvulsant)\%}

API (hypotensive)\%

API (muscle relaxant)\%

Transdermal Base (Example: Lipoderm) (active transport)

Table 4: Writing a compounded transdermal pain prescription.

Potential for lower systemic absorption and minimization of side effects: Pain creams are typically applied at the site of pain. There have been a number of studies focusing on the differences in systemic absorption and incidences of side effects between oral and transdermal NSAIDs. In an ex vivo study of patients suffering with osteoarthritis of the knee, high-dose topical ibuprofen (1125 mg via $22.5 \mathrm{~g}$ of $5 \%$ ibuprofen gel) was shown to reach therapeutic concentrations in the target muscles with low concentrations in the synovial fluid and plasma [41]. Similarly, another study found that topical NSAID administration led to a maximal plasma concentration of less than $15 \%$ than that resulting from oral NSAID administration [41]. The use of transdermal ketoprofen in patients with rheumatic diseases or trauma was shown to lead to high tissue concentrations of drug with low serum levels, leading to a low incidence of systemic side effects [42]. In addition, Finch et al. discovered that plasma concentrations of topical $10 \%$ ketamine were negligible when measured 1 hour after administration to patients with complex regional pain syndrome for the treatment of allodynia while still inhibiting the symptoms of allodynia in stimulated symptomatic limbs compared to placebo [43]. These findings all suggest lower systemic absorption of the pain medications when administered in their topical formulations compared to oral forms [44].

Transdermal pain medication may reduce some of the side effects commonly reported with oral pain medication, potentially through the reduction in systemic absorption of the drug. A systematic review of adverse events in clinical trials comparing topical and oral NSAIDs showed a slightly lower number of systemic adverse events and withdrawals due to adverse events in the topical group than the oral group [41]. Another study by McMahon et al. revealed that the risk of GI complications is minimal with exposure to topical NSAIDs as compared to oral NSAIDs using a record-linkage study [45]. In this study, an initial suspected association between topical NSAIDs and GI side effects was observed due to the confounding effects of concomitant use of oral NSAIDs and ulcer-healing drugs. When analyses were adjusted for these confounding effects, there was no significant association between topical NSAID use and GI adverse events. A study by Underwood et al. of elderly patients with chronic knee pain showed that oral and topical NSAIDs had similar efficacy; however, oral NSAIDs were found to lead to more minor adverse events than topical versions, leading this team of researchers to recommend topical NSAIDs over oral for treating elderly patients with chronic musculoskeletal pain [41].

Convenience and potential for better adherence to treatment regimen: Transdermal compounded pain medication can be administered directly to the site of pain, providing the patient with a simple and convenient treatment regimen. Because multiple APIs can be combined in a single, customized product, the need for multiple medications potentially with multiple daily doses at different times throughout the day can be avoided. This simplification may lead to better patient adherence to treatment regimens and better overall treatment outcomes [31]. In addition, this method of drug delivery can be especially helpful for patient populations that have issues with the administration of oral medication, such as those who have difficulty swallowing pills.

Minimizing the risk of abuse and addiction: The use of transdermal compounded pain medication as an alternative to oral pain medication can be a highly beneficial tool in the treatment of patients with risk of abuse and addiction to oral pain medication. Transdermal compounds can often help effectively manage pain without the need for potentially addictive narcotics or other oral treatment options. Highlighting this benefit, Christiansen and White present a case study of a patient's treatment for musculoskeletal pain with a transdermal compound from the perspective of both the patient and compounding pharmacist [46]. The patient (Christiansen) suffered from chronic musculoskeletal pain as well as struggled with prescription drug addiction, leading him to turn to compounding pharmacist (White). A custom transdermal compounded medication was prepared for the patient containing $20 \%$ ketoprofen and $10 \%$ lidocaine in Lipoderm ${ }^{\circ}$ applied every 2 to 8 hours, as needed. The patient reported a decrease in overall pain from a 10 to a 2 on a 1 to 10 relative pain scale upon using this treatment without triggering any of his drug addiction issues. Seven years later, the treatment began to be ineffective for the patient, so the compound was modified to also include $2 \%$ cyclobenzaprine, which helped to manage the patient's increasing muscle cramping and relieve his pain, thus, providing him with an effective, long-term treatment option without contributing to the patient's struggle with drug addiction.

Compounded Transdermal Medications for Various Types of PainCase Studies and Clinical Trials: Awareness and prescribing of compounded transdermal medication for pain relief have increased dramatically in recent years. A recent survey of physicians found that $81 \%$ have prescribed topical medication for the treatment of musculoskeletal pain, and $63 \%$ have prescribed topical medication for the treatment of neuropathic pain [47]. Because pain management requires tailoring to the unique needs of each individual patient, the use of compounded transdermal medication in pain management plans can provide patients with customized medication options. In the following section, we provide recent examples as evidence for the success of compounded transdermal medication in the treatment of a number of different pain-related conditions.

Neuropathic pain is often very difficult to treat, and prescribers have utilized various combinations of compounded transdermal preparations when treating patients. In a long-term, open-label clinical trial conducted by Lynch et al., the effect of a compounded $2 \%$ amitriptyline and $1 \%$ ketamine cream was examined for the treatment of neuropathic pain for a mixed population of patients, including those suffering from diabetic neuropathy, postherpetic neuralgia, or postsurgical/posttraumatic neuropathic pain [48]. The study evaluated patients over a period of 12 months. A 34\% average reduction in pain was reported in patients over the first 6 months of the study, while a $37 \%$ average reduction of pain was reported over 12 months. At the end of the 12 -month study, $40 \%$ of the patients reported a $50 \%$ or greater reduction in their overall pain. Few adverse events were reported in the study, none of which were considered serious events related to the study drug.

Barton et al. described the successful use of transdermal pain medication in the treatment of chemotherapy-induced neuropathy in cancer patients [49]. In this placebo-controlled study, the efficacy of a compounded transdermal formulation of baclofen, amitriptyline $\mathrm{HCl}$, and ketamine was evaluated compared to placebo in 208 patients over 
a 4-week period. A sensory subscale was used to evaluate efficacy in this trial. A greater improvement in both sensory and motor subscales among patients randomized to study drug over placebo was observed. Improvements included reduced tingling, cramping, and shooting/ burning pain in the hands. No significant differences in toxicities were observed between the 2 study arms, and most adverse events were mild in severity.

In another study by Tam and Furlan, a compound with the combination of lidocaine and ketamine for the treatment of neuropathic pain was analyzed in a retrospective chart review [50]. Twenty-one patients treated with 2 to $10 \%$ lidocaine/5 to $10 \%$ ketamine in PLO or Lipoderm ${ }^{\star}$ for diagnosed or suspected neuropathic pain were identified in this review. In some cases, the lidocaine/ketamine combination was compounded with other APIs, including ketoprofen, gabapentin, amitriptyline, and nifedipine. Of the patients who had records of followup after treatment, $83 \%$ were reported to have benefited from treatment with the cream, and only 2 patients discontinued treatment due to adverse events relating to skin reactions.

Another painful condition with potential for substantial benefit from compounded transdermal medication is arthritis, which accounts for a large portion of adult pain sufferers and affects more than $21 \%$ of the US population [51]. Marshall et al. reported a series of individual case studies in which custom compounded transdermal preparations were successfully utilized in treating patients suffering from arthritisrelated joint pain [51]. Various formulations of transdermal creams or gels applied directly the site of pain were used, and patients reported effective pain management without adverse reactions that was often better than that with their previously prescribed oral pain medication regimens. For example, an elderly woman with difficulty controlling rheumatoid arthritis pain in the neck and shoulder was given a transdermal cream was prepared using $6 \%$ ketamine, 10 to $15 \%$ ketoprofen, and 4 to $6 \%$ lidocaine in Lipoderm ${ }^{\circ}$, with instructions to apply the cream to the areas of pain every 4 to 6 hours. The patient reported pain relief within 30 to 60 minutes after application and continued to use the custom prescription at the time of publication with no reported adverse events. While the evidence in this report was presented as a series of individual case studies, the authors stated that these examples represent typical formulations used for the treatment of arthritis-related pain [51].

Additionally, Varadi et al. performed a clinical study to evaluate the use of a transdermal formulation of $10 \%$ ibuprofen for the treatment of moderate-to-severe osteoarthritis of the knee [52]. In this 2-week, placebo-controlled trial, efficacy was evaluated using common methods of pain assessment, including the Western Ontario and McMaster Universities (WOMAC) Osteoarthritis Index and the Visual Analog Scale (VAS). Patients treated with transdermal ibuprofen had positive clinical improvements for all efficacy endpoints, which were superior to the results observed in the patients randomized to placebo. No significant adverse events were noted in this study.

Transdermal pain treatment preparations have also been successful in the management of outpatient postoperative pain, a particularly challenging area of pain management. As described earlier, healthcare providers are becoming more cautious about prescribing oral opioids but have limited options in the prescription of effective painkillers that patients can self-administer at home to allow them to limit their hospital stays following surgery. Alexander and Wynn reported a case study of the successful treatment of a patient following gall bladder removal with a compounded combination of $6 \%$ gabapentin,
$2 \%$ amitriptyline, $5 \%$ lidocaine, and $10 \%$ ketoprofen in Lipoderm ${ }^{\circ}$ following problems with previous regimens of oral Vicodin and lidocaine patches [53]. The authors reported that transdermal preparations of NSAIDs, local anesthetics, anticonvulsants, and tricyclic antidepressants alone or in combination have also proven useful in the treatment of postoperative pain.

While the use of compounded transdermal medication has been shown to be effective in the treatment of common pain conditions, such as neuropathic and arthritis-related pain, these treatment options may be particularly important in the treatment of less common pain conditions that may not have standard treatment options.

In a retrospective study of 51 patients, Boardman et al. evaluated the effect of transdermal gabapentin 2 to $6 \%$ in Lipoderm ${ }^{\circ}$ for the treatment of vulvodynia, a vaginal condition affecting nearly 14 million women in the US alone [54]. There is no clear standard of care for this condition; however, typical treatment involves oral tricyclic antidepressants and anticonvulsants, which have been shown to have limited efficacy and adverse effects, including sedation, dizziness, and dry mouth. In this clinical trial, of the 35 evaluable patients who were treated for 8 weeks or longer, $28(80 \%)$ reported at least $50 \%$ or greater improvement in pain scores. In addition, among patients with localized vulvodynia, 17 of 20 patients reported improved sexual function following treatment. Overall, there were minimal adverse events associated with this treatment, making it a viable alternative treatment option for a condition lacking an effective standard of care.

In another example, Lehman and Sciallis reported a case study of a patient with a long history of chronic, idiopathic proctodynia, a pain syndrome affecting the perianal region, for which an effective standard treatment option could not be identified [55]. The patient had been treated with topical $1 \%$ hydrocortisone and zinc oxide for approximately 20 years, but the pain had become intense, and this treatment was no longer effective. Gabapentin $(300 \mathrm{mg}$ daily, later increased to $600 \mathrm{mg}$ three times daily) was then prescribed; however, the effectiveness of the treatment was only temporary. Therefore, a combination of $2.5 \%$ amitriptyline and $0.5 \%$ ketamine in a compounded cream was prescribed for topical application and found to effectively relieve this patient's pain after just 2 days of treatment. The patient was able to discontinue hydrocortisone treatment and taper down his gabapentin administration. After 1 year of the compounded treatment regimen, his pain remained under control, resulting in a significantly improved quality of life.

\section{Conclusion}

Compounded transdermal medications have been shown to be effective in the treatment of neuropathic, musculoskeletal, arthritic, and postoperative pain, among other pain-related conditions, and they continue to be explored as a viable alternative option for the management of pain related to a variety of conditions. Compounded transdermal medications effectively reduce pain symptoms while offering a decreased risk of adverse effects commonly associated with oral pain medication, and they may help decrease the risk of drug abuse and addiction. Compounded transdermal preparations for pain management can provide healthcare providers with individualized and potentially more effective options for the treatment of both acute and chronic pain-related conditions. 


\section{Conflicts of Interest}

The authors are employees of PCCA, the manufacturer of Lipoderm ${ }^{\oplus}$ and supporter of the study.

\section{Acknowledgment}

The authors acknowledge Lisa Ambrosini Vadola, PhD for her assistance with the preparation of this manuscript.

\section{References}

1. Institute of Medicine (2011) Relieving pain in America: A blueprint for transforming prevention, care, education, and research. The National Academies Press, Washington, DC.

2. National Center for Health Statistics (2006) Health, United States, 2006 with Chartbook on Trends in the Health of Americans. Hyattsville, MD.

3. National Institutes of Health (2010) Pain management fact sheet.

4. Centers for Disease Control and Prevention (2014) Opioid painkiller prescribing: where you live makes a difference.

5. Cleveland Clinic (2008) Acute vs. chronic pain.

6. Waghmarae R, Lelito R, Detschner A, Salcedo D (2013) Poor adherence to opioid pain management regimens. Practical Pain Management.

7. Harirforoosh S, Asghar W, Jamali F (2013) Adverse effects of nonsteroidal anti-inflammatory drugs: an update of gastrointestinal, cardiovascular, and renal complications. J Pharm Pharm Sci 16: 821-847.

8. Conaghan PG (2012) A turbulent decade for NSAIDs: update on current concepts of classification, epidemiology, comparative efficacy, and toxicity. Rheumatol Int 32: 1491-1502.

9. Perneger TV, Whelton PK, Klag MJ (1994) Risk of kidney failure associated with the use of acetaminophen, aspirin, and nonsteroidal antiinflammatory drugs. N Eng J Med 331: 1675-1679.

10. Hauser RA (2010) The acceleration of articular cartilage degeneration in osteoarthritis by nonsteroidal anti-inflammatory drugs. J Prolotherapy 2: 305-322.

11. Mazer M, Perrone J (2008) Acetaminophen-induced nephrotoxicity: pathophysiology, clinical manifestations, and management. J Med Toxicol 4: 2-6.

12. Larson AM, Polson J, Fontana RJ, Davern TJ, Lalani E, et al. (2005) Acetaminophen-induced acute liver failure: results of a United States multicenter, prospective study. Hepatology 42: 1364-1372.

13. Swegle JM, Logemann C (2006) Management of common opioidinduced adverse effects. Am Fam Physician 74: 1347-1354.

14. US Food and Drug Administration (2011) FDA limits acetaminophen in prescription combination products; requires liver toxicity warnings.

15. See S, Ginzburg R (2008) Choosing a skeletal muscle relaxant. Am Fam Physician 78: 365-370.

16. Clivatti J, Sakata RK, Issy AM (2009) Review of the use of gabapentin in the control of postoperative pain. Rev Bras Anestesiol 59: 87-98.

17. Dworkin RH, O'Connor AB, Audette J, Baron R, Gourlay GK, et al. (2010) Recommendations for the pharmacological management of neuropathic pain: an overview and literature update. Mayo Clin Proc. 85: S3-S14.

18. Guay DRP (2001) Adjunctive agents in the management of chronic pain. Pharmacotherapy 21: 1070-1081.

19. Centers for Disease Control and Prevention (2010) Unintentional drug poisoning in the United States.

20. United States Department of Health and Human Services (2013) Prescribers with questionable patterns in Medicare Part D.

21. Centers for Disease Control and Prevention (2014) Prescription drug overdose in the United States: Fact Sheet.

22. United States Department of Health and Human Services (2013) Addressing prescription drug abuse in the United States: current activities and future opportunities.
23. Birnbaum HG, White AG, Schiller M, Waldman BA, Cleveland JM, et al. (2011) Societal costs of prescription opioid abuse, dependence, and misuse in the United States. Pain Med 12: 657-667.

24. Ryder S-A, Stannard CF (2005) Treatment of chronic pain: antidepressant, antiepileptic and antiarrhythmic drugs. Continuing Education in Anaesthesia. Critical Care and Pain 5: 18-21.

25. World Health Organization (2003) Adherence to long-term therapies: evidence for action. Geneva, Switzerland.

26. Claxton AM, Cramer J, Pierce C (2001) A systematic review of the associations between dose regimens and medication compliance. Clin Therapeutics 23: 1296-1310.

27. Osterberg L, Blaschke T (2005) Adherence to medication. N Engl J Med 353: 487-497.

28. Mafi JN, McCarthy EP, Davis RB, Landon BE (2013) Worsening trends in the management and treatment of back pain. JAMA Intern Med 173: 1573-1581.

29. Radnofsky L, Walker J (2014) DEA restricts narcotic pain drug prescriptions. Wall Street Journal.

30. MacQuarrie B (2014) Blue Cross cuts back on painkiller prescriptions. The Boston Globe.

31. Paudel KS, Milewski M, Swadley CL, Brogden NK, Ghosh P, et al. (2010) Challenges and opportunities in dermal/transdermal delivery. Ther Deliv 1: 109-131.

32. Pharmaceutical Compounding Expert Committee (2014) Pharmaceutical compounding-nonsterile preparations. In: United States Pharmacopeia Chapter 795 (Revision). Rockville, MD: United States Pharmacopeial Convention.

33. Krochmal L (2009) Considerations before choosing (extemporaneously) compound products. Dermatol Ther 22: 225-228.

34. Allen LV (2003) Contemporary pharmaceutical compounding. Ann Pharmacother 37: 1526-1528.

35. PCCA (2014) PCCA Education.

36. Murdan S (2005) A review of pluronic lecithin organogel as a topical and transdermal drug delivery system. Hospital Pharmacist 12: 267-270.

37. PCCA (2013) Lipoderm

38. PCCA (2011) PCCA Lipoderm ${ }^{\circ}$ and ketoprofen transdermal study.

39. PCCA (2011) PCCA Lipoderm ${ }^{\circ}$ delivers four drugs simulataneously in transdermal study.

40. PCCA How to Write a Compounded Pain Prescription. Document 98742.

41. Underwood M, Ashby D, Carnes D, Castelnuovo E, Cross P, et al. (2008) Topical or oral ibuprofen for chronic knee pain in older people. The TOIB study. Health Technol Assess 12.

42. Jorge LL, Feres CC, Teles VEP (2011) Topical preparations for pain relief: efficacy and patient adherence. J Pain Res 4: 11-24.

43. Finch PM, Knudsen L, Drummond PD (2009) Reduction of allodynia in patients with complex regional pain syndrome: a double-blind, placebocontrolled trial of topical ketamine. Pain 146: 18-25.

44. Author's Note: The authors acknowledge there are many variables associated with transdermal bases, pharmacokinetics, and drugs; therefore it is advised that the prescriber work closely with pharmacists and pharmacies that have significant experience with compounded transdermal pain compounds for proper advisement on appropriate dosing regimens.

45. McMahon AD, McGilchrist MM, White G, Murray FE, McDevitt DG, et al. (1995) Topical non-steroidal anti-inflammatory drugs and admission to hospital for upper gastrointestinal bleeding and perforation: a record linkage case-control study. BMJ 311: 22-26.

46. Christiansen J, White L (2010) Unique challenges in compounding: managing severe musculoskeletal pain in a recovered substance abuser. Int J Pharm Compd 14: 311-314.

47. Warner M, Tuder D (2014) A brief survey on prescriber beliefs regarding compounded topical pain medications. Int J Pharm Compd 18: 182-188. 
Citation: Branvold A, Carvalho M (2014) Pain Management Therapy: The Benefits of Compounded Transdermal Pain Medication. J Gen Practice 2: 188. doi:10.4172/2329-9126.1000188

Page 8 of 8

48. Lynch ME, Clark AJ, Sawynok J, Sullivan MJ (2005) Topical amitriptyline and ketamine in neuropathic pain syndromes: an open-label study. J Pain 6: 644-649.

49. Barton DL, Wos EJ, Qin R, Mattar BI, Green NB, et al. (2011) A doubleblind, placebo-controlled trial of a topical treatment for chemotherapyinduced peripheral neuropathy: NCCTG trial N06CA. Support Care Cancer 19: 833-841

50. Tam E, Furlan AD (2012) Transdermal lidocaine and ketamine for neuropathic pain: a study of effectiveness and tolerability. Open Neurol J 6: 58-64.

51. Marshall R, Vidaurri VA, Boomsma D, Buchta AJ, Vail J (2008) Case reports: compounding to relieve arthritis pain. Int J Pharm Compd 12: 498-504.
52. Varadi G, Zhu Z, Blättler T, Hösle M, Loher A, et al. (2013) Transdermal ibuprofen for moderate to severe knee osteoarthritis. Pain Physician 16: E749-E762.

53. Alexander K, Wynn T (2007) Transdermal gel in the treatment of postoperative pain. Int J Pharm Compd 11: 181-184.

54. Boardman LA, Cooper AS, Blais LR, Raker CA (2008) Topical gapapentin in the treatment of localized and generalized vulvodynia. Obstet Gynecol 112: 579-585.

55. Lehman JS, Sciallis GF (2008) Effective use of topical amitriptyline hydrochloride $2.5 \%$ and ketamine hydrochloride $0.5 \%$ for analgesia in refractory proctodynia. J Drugs Dermatol 7: 887-889. 